\title{
EFFECTIVENESS OF ADDITIONAL THERAPY WITH NSAID (AULIN) ON DISTRIBUTION OF SHALLOW AND DEEP PERIODONTAL POCKETS IN PATIENTS WITH CHRONIC PERIODONTITIS (Pilot study)
}

\author{
Chr. Popova, A. Mlachkova \\ Department of Periodontology, Faculty of Dental Medicine, \\ Medical University of Sofia
}

\begin{abstract}
Introduction: Currently accepted understanding about the role of the host inflammatory response in the progression of destructive periodontal diseases is the basis of therapeutic approaches with NSAIDs. Reduced levels of IL-1 and $\mathrm{PGE}_{2}$ in crevicular fluid and gingival tissues are measured in patients with adjunctive host modulation therapy in comparison with conventional mechanical therapy alone in numerous studies. The healing in patients with this additional therapy demonstrates successful elimination of gingival inflammation with pocket depth reduction and attachment level improvement.

Aim: To investigate the effectiveness of additional host modulation therapy with NSAID (Aulin) in patients with chronic periodontitis.

Material and methods: The study involves 14 adult patients suffering moderate to advanced periodontitis, instructed for strict daily plaque control (including personal hygiene involving interdental brushes and dental floss) and antimicrobial mouth rinsing with $0.2 \%$ chlorhexidine (Corsodyl) and is treated with scaling and root planning. On the 5th day of the treatment the patients are put on adjunctive systemic administration of NSAID (Aulin) for 14 days, twice daily $x 100 \mathrm{mg}$. The efficiency of the therapy is measured at its 6 th week with the same clinical parameters - PD, CAL, HI, and PBI.

Results: The results of the treatment depict significant shift in the distribution of shallow and deep periodontal pockets. The shallow pockets $(1-3 \mathrm{~mm})$ in the initial status of the patients are $23.8 \%$ from all measured pockets and at the end of the applied treatment this number rises up to $75.7 \%$. The initial level of the deep periodontal pockets $(\geq 5 \mathrm{~mm})$ is $9.8 \%$ and decreases to $0.5 \%$ after treatment. The quantity of pockets with moderate depth (3$5 \mathrm{~mm}$ ) decreases from $66.5 \%$ to $38.7 \%$ at the end of the evaluated treatment.
\end{abstract}

Conclusion: Within the limits of this pilot study we may conclude that the additional host modulation therapy with Aulin may enhance the outcomes of nonsurgical therapy in patients with chronic periodontitis.

Key words: chronic periodontitis, proinflammatory cytokines, nonsurgical therapy, NSAIDs, pocket depth

The understanding of the pathogenesis of periodontitis has evolved from a purely plaque-associated disease to the current thinking, which places a renewed emphasis on the host's response to the bacteria. The etiology of the periodontal diseases is a bacterial infection but it is the host's reaction to the presence of bacteria that mediates tissue destruction. While it is apparent that plaque is essential for the development of the disease, the severity and pattern of the disease are not explained solely by the amount of plaque present. Recently we have come to believe that the production of pro-inflammatory mediators from gingival tissues aggravate the destruction of soft and hard periodontal tissues whose clinical expression is the severity of periodontal disease $(1,8,10)$

Recently available data on the role of the host in the inflammatory response and progression of destructive periodontal diseases is the basis of therapeutic approaches by means of inhibition of the proinflammatory mediators that are involved in destructive tissue response $(4,6)$. Considerable numbers of investigations demonstrate a trend toward successful reduction of proinflammatory cytokines in GCF/gingiva and reduced levels of tissue inflammation as a result of the use of NSAIDs in the course of nonsurgical periodontal therapy. Reduced levels of IL-1 and $\mathrm{PGE}_{2}$ in crevicular fluid and gingival tissues are measured in patients with host modulation therapy during adjunctive NSAIDs in comparison with conventional mechanical therapy $(2,3)$. The healing in patients with this additional therapy demonstrates successful elimination of gingival inflammation with pocket depth reduction and attachment level improvement.

Conducted clinical study demonstrates the efficacy of the NSAID in the treatment and control of progressive 
periodontal disease. NSAID suppress gingival inflammatory response and inhibit bone destruction $(3,7,9)$. The initiation of periodontal diseases is associated with hyper production of cytokines and other mediators of the inflammatory reaction such as: TNF- $\alpha$, IL-1- $\beta$, IL- 6 and e.t. The high levels of cytokines are related to the progression of the diseases; the active phase of periodontal diseases is characterized with low levels of tissues inhibitors of MMP and high levels of $P G$, secreted from polymorfonuclear neutrofils, fibroblasts, macrophages and lymphocytes. There is evidence that NSAID have effect over inflammatory status of the periodontal tissue decreasing the prostaglandin and cytokine concentration $(3,5,6)$.

This study aims to investigate the effectiveness of additional host modulation therapy with NSAID (Aulin) in distribution of shallow and deep periodontal pockets in patients with chronic periodontitis.

\section{MATERIALS AND METHODS}

The study involves 14 adult patients suffering moderate to advanced periodontitis, diagnosed clinically and radiographically. Subjects are clear of systemic diseases and treatment within 6 months. The initial status and healing response to therapy is evaluated by full mouth pockets depth (PD) and clinical attachment level (CAL) measurements. HI is used for plaque control assessment in the course of the treatment. PBI is applied to evaluate the degree of inflammation and the quantity of bleeding sites before treatment and to measure the elimination of the inflammation at the end of conventional therapy with adjunctive Aulin. All patients are instructed for strict daily plaque control (including personal hygiene involving interdental brushes and dental floss) and antimicrobial mouth rinsing with $0.2 \%$ chlorhexidine (Corsodyl) and are treated with scaling and root planning. Close periodontal curettage is performed in pockets deeper than $5 \mathrm{~mm}$ with persistent bleeding on probing during the initial therapy. On the 5th day of the treatment the patients are put on adjunctive systemic administration of NSAID (Aulin) for 14 days, twice daily $x 100 \mathrm{mg}$. The efficiency of the therapy is measured at its 6th week with the same clinical parameters - PD, CAL, HI, and PBI.

\section{RESULTS}

The results of the treatment depict significant shift in the distribution of shallow and deep periodontal pockets. The shallow pockets $(1-3 \mathrm{~mm})$ in the initial status of the patients are $23.8 \%$ from all measured pockets and at the end of the applied treatment this number rises up to $75.7 \%$. The initial level of the deep periodontal pockets $(\geq 5 \mathrm{~mm})$ is $9.8 \%$ and decreases to $0.5 \%$ after treatment. The quantity of pockets with moderate depth $(3-5 \mathrm{~mm})$ decreases from $66.5 \%$ to $38.7 \%$ at the end of the evaluated treatment.

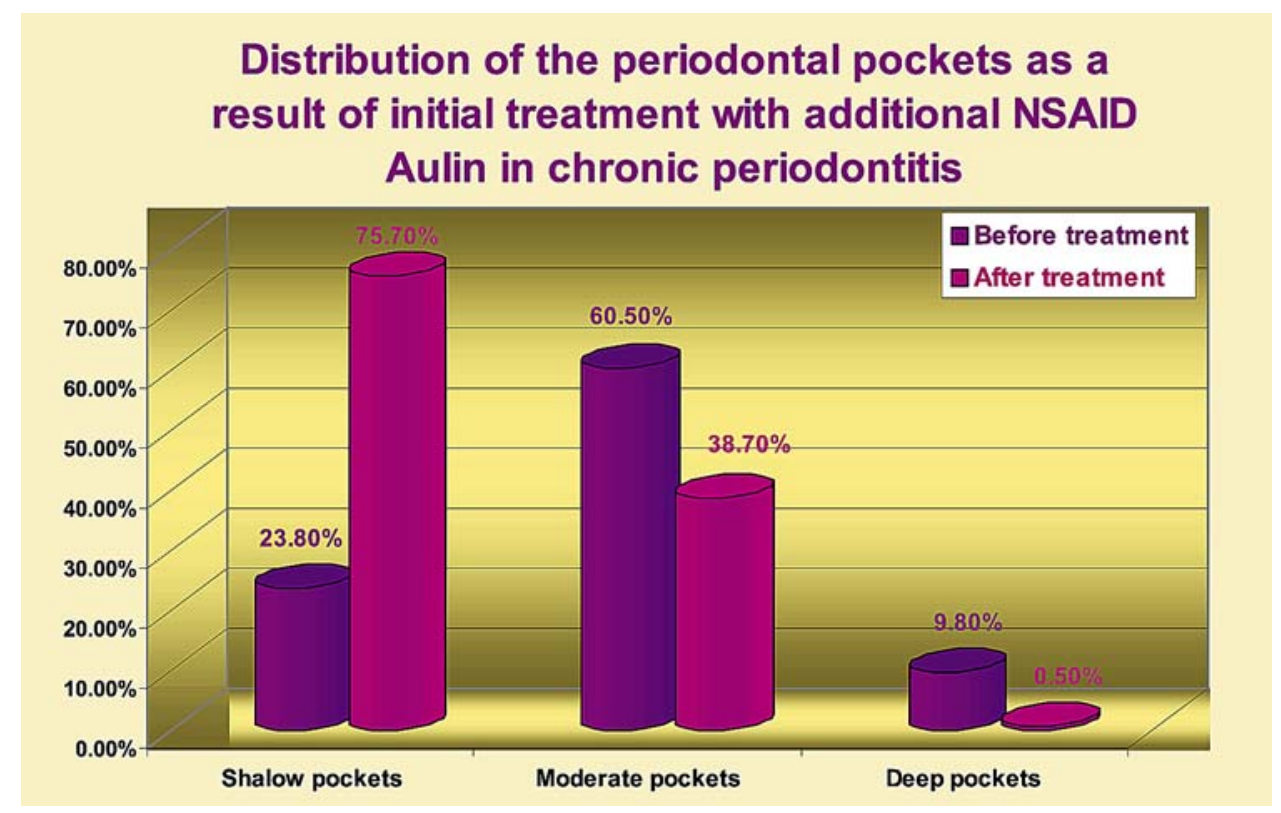

Tabl. 1. 


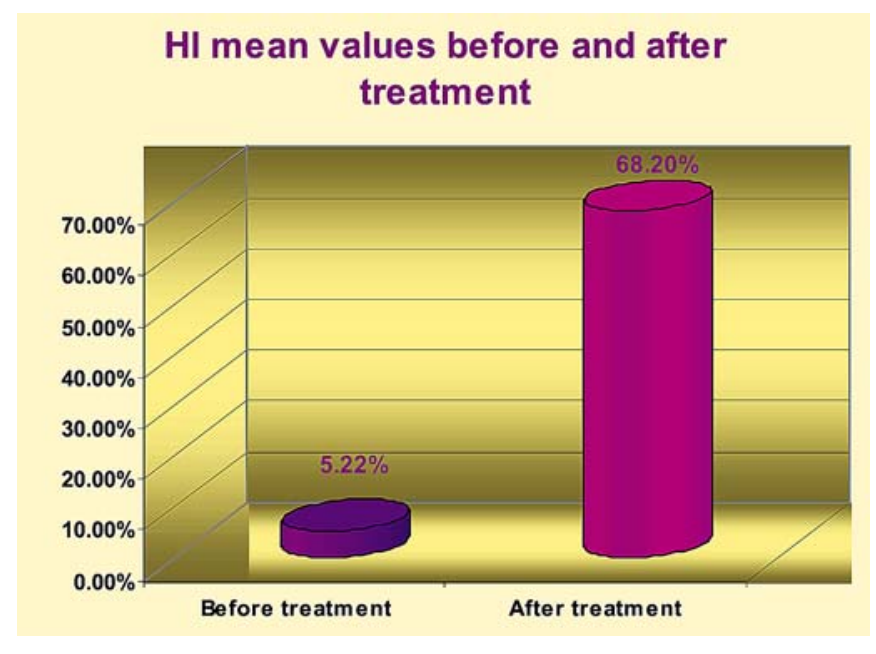

\section{CONCLUSION}

Within the limits of this pilot study we may conclude that the additional host modulation therapy with Aulin may enhance the outcomes of nonsurgical therapy in patients with chronic periodontitis. The reduction of the amount of deep periodontal pockets in the study suggests that the adjunctive administration of NSAID may diminish the need of resective periodontal pocket treatment. The established periodontal status as a result of the treatment designates periodontal environment presuming successful periodontal maintenance.

Tabl. 2.

\section{REFERENCES:}

1. Mlachkova A. Administration of nonsteroid anti-inflammatory medication in treatment of periodontitis. Zabolekarski pregled 2004; 1:58-66.

2. Bennett A., G.Villa. Nimesulide: an NSAID that preferentially inhibits COX2 , and has various unique pharmacological activities. Exp. Opin.Pharmacother.2000; 1:277-284.

3. Famaey JP. In vitro and in vivo pharmacological evidence of selective cyclooxygenase- 2 inhibition by nimesulide: an overview. Inflamm Res 1997; 46(11): 437-46.

4. Nassar C., P. Nassar, L. Spolidorio. Selective cyclooxygenase-2 inhibition prevents bone resorption. Braz Oral Res
2005; 19(1):36-40.

5. Taniguchi Y., K. Yokoyama, T. Nakamura, A. Toda, H. Shimeno. Inhibitions by nimesulide of prostaglandin production in rat macrophages. Drugs Exp Clin Res 1998; 24(1):17-27.

6. Tipton D., J. Flin, M. Dabbous. Cyclooxygenase-2 inhibitors decrease interleukin- $1 \alpha$-stimulated prostaglandin E2 and IL-6 production by human gingival fibroblasts. J Periodontol 2003; 74; 1754 1763.

7. Vardar-Sengыl S., H. Baylas, A. Huseyinov. Effect of selective cyclooxygenase- 2 inhibition on gingival tissue levels of prostaglandin E2 and prostaglandin F 26 and clinical parameters of chronic periodontitis. J Periodontol 2003; 74: 57-63

8. Salvi E., P. Lang. The effects of nonsteroidal anti-inflammatory drugs (selective and non- selective) on the treatment of periodontal diseases. Current Pharmaceutical Design, vol.11; 2005:1757-1769.

9. Waite I. M., C. A. Saxton, A. Young, B. J. Wagg and M. Corbett. The periodontal status of subjects receiving non-steroidal anti-inflammatory drugs. J Periodontal Research 16, 100-108; 1981.

10. O'Hehir T. Host response modulation: a promising new addition to periodontal disease management. The Journal of Practical Hygiene, 1999; July/ August: 25-31.

\section{Address for correspondence:}

Assoc. prof. Christina Popova, PhD

Department of Periodontology, Faculty of Dental Medicine,

Medical University of Sofia,

1, Georgi Sofiiski Str., Sofia, Bulgaria

Mobile: +359 8887590 49;

E-mail: hrpopova@yahoo.com

**Antoaneta Mlachkova - Assist. Prof. in the Department of Periodontology, Faculty of Dental Medicine, Medical University - Sofia

E-mail: dr_mlachkova@abv.bg 